\title{
Loop shaping of multivariable systems with hard constraints on the control signal
}

\author{
Wolfgang Reinelt \\ Department of Electrical Engineering \\ Linköping University, S-581 83 Linköping, Sweden \\ WWW: http://wwW. control.isy.liu.se/ ${ }^{\sim}$ wolle/ \\ Email: wolle@isy.liu.se
}

April 24, 2001

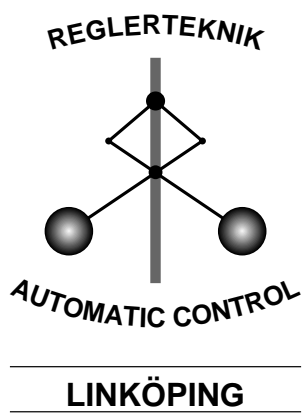

Report no.: LiTH-ISY-R-2320

Technical reports from the Automatic Control group in Linköping are available by anonymous ftp at the address ftp.control.isy.liu.se. This report is contained in the portable document format file 2320.pdf. 


\title{
Loop shaping of multivariable systems with hard constraints on the control signal*
}

\author{
Wolfgang Reinelt \\ Department of Electrical Engineering, Linköping University, 58183 Linköping, Sweden. \\ E-mail: wolle@isy.liu.se, Fax: +4613282622.
}

\begin{abstract}
A general framework for the design of multivariable control systems subject to hard constraints on each control channel is developed. The design procedure is based on the $\mathcal{H}_{\infty}$ Loop Shaping and relies on the calculation of the maximum possible control amplitude for a class of reference signals, bounded in amplitude and rate. Special attention is given to adjustment of the design weights in order to meet prescribed bounds on each control signal. A simulation example, the control of the vertical dynamics of an aircraft, illustrates the suggested procedure.
\end{abstract}

Keywords: Constraint Control, Saturation Avoidance, Hard Bounds, Rate Constraints, Multivariable Systems.

\section{Introduction and Motivation}

Most practical control problems are dominated by hard bounds. Valves can only be operated between fully open and fully closed, pumps and compressors have a finite throughput capacity and tanks can only hold a certain volume. These input- or actuator-bounds convert the linear model into a nonlinear one. Exceeding these prescribed bounds causes unexpected behaviour of the system - large overshoots, low performance or (in the worst case) instability. A classical example for the detrimental effect of neglecting constraints is the Chernobyl nuclear power plant disaster in 1986. Therefore, design of controllers for systems with hard constraints is a quite vivid area of research, see for example the recent books $[1,2]$ or special issues $[3,4]$ and the references therein. The problem has been addressed within the Model Predictive Control community, see [5], and another popular approach is to use so-called Anti Windup Bumpless Transfer schemes, see [6, 7] for instance.

Controller designs that encounter the saturation effect a-priori are usually separated into two categories: (1) designs that prevent saturation of the control signal and therefore enjoy a linear framework (as long as plant and controller are linear) and (2) methods that allow saturation and are therefore facing a nonlinear setup. In the second case, analysis (in terms of stability, controllability and feasibility) of this nonlinear system is discussed in $[8,9,10]$. Design schemes that handle saturations using a (nonlinear) control law have recently been proposed in [11, 12] for instance.

\footnotetext{
*A preliminary version of this work has been presented at the 1999 International Symposium on QFT and Robust Frequency Domain Methods, Durban, South Africa and some parts have been published as a PhD dissertation at Paderborn University, Paderborn, Germany. This paper has not been submitted to or published in another journal.
} 
This work employs the first - saturation avoiding - philosophy. In the case of SISO systems, avoiding the saturation generally makes time responses dull, which is not a priori clear in the case of multivariable systems, due to interactions between the different in- and output channels. This makes it worthwile investigating the problem of designing linear controllers for multivariable systems. Moreover, the simulation example will demonstrate that switching to a saturation avoiding controller will of course enhance performance, compared to a linear controller that runs into saturation.

To solve the constraint control problem, one implicitly has to restrict the amplitude of all external signals - independent of the technique used in particular. Our approach, however, poses an additional restriction on the rate of the external signals. In many practical situations, this is a more accurate description (than without rate restriction) of all external signals, possibly arising during runtime: in the example of the tank from above, not only the liquid-level is bounded (by the tanks height), additionally the liquid cannot change its level arbitrarily fast. Therefore, a design, directly based on this description will avoid a conservative control system, which has been demonstrated in related works, using this approach [13, 14, 15].

The design procedure suggested here focuses on the adjustment of weights in $\mathcal{H}_{\infty}$ Loop Shaping, so that a certain prescribed bound is met. This will lead us to a user-interactive design procedure, where the engineer still can incorporate experience on the choice of weights form the "classical" $\mathcal{H}_{\infty}$ Loop Shaping. This approach can also be regarded as systematically adjusting an existing linear controller, which is not explicitely designed with the bound on a control signal to a controller, that guarantees not to saturate (for a prescribed set of reference signals). A somewhat related line of research, based on these assumptions on external signals, studies the design of optimal single-input single-output control systems with respect to hard constraints have been studied in [16] or robust and constrained systems, see [17]. The main technique used there is a (double-) Youla parameterisation to obtain a computationally feasible problem. These approaches are (so far) restricted to single-input single-output systems.

Paper outline: Sec. 2 discusses the calculation of the maximum possible amplitude of the control signal in a multivariable system. Then, Sec. 3 presents $\mathcal{H}_{\infty}$ Loop Shaping, extended for the design of systems with bounded control signals. The crucial point within the Loop Shaping Design Procedure is the systematic adjustment of the design weights. In our case the task becomes even more difficult, when the prescribed hard bounds (on the control signal) are not met. We present a general guideline in Sec. 4. The proposed method is then illustrated with a multivariable example in Sec. 5, the control of the vertical dynamics of an aircraft. We summarise in Sec. 6 .

\section{Multivariable Systems and their Maximum Control Amplitude}

As motivated in the introduction, we study multivariable control systems with reference signals, bounded in amplitude and speed. Aim of the controller design is to handle hard bounds of the control signal. The setup is given in Fig. 1 and we will denote the transfer function from reference to control signal by $H=(I-K G)^{-1} K$, and by $h$ its impulse response. We give the following definitions, which are straightforward extensions of those given by [13] to the multivariable case:

Definition 1 (Admissible Reference Signal) Let $0 \preceq R, \dot{R} \in \mathbb{R}^{n}$. A (vector-valued) continuous and piecewise differentiable ${ }^{1}$ reference signal $r$, fulfilling $r(t)=0$ for all $t \leq 0$, is called $(R, \dot{R})$-admissible, when the following properties hold:

\footnotetext{
${ }^{1}$ this allows a countable number of time stamps, where $r$ is not differentiable.
} 
1. $|r(t)| \preceq R$ for all $t>0$ and

2. $|\dot{r}(t)| \preceq \dot{R}$ for all $t>0$,

whereas $\preceq$ denotes component-wise $\leq$ and $|\cdot|$ has to be evaluated component-wisely in this context. The set of all $(R, \dot{R})$-admissible reference signals is denoted by $\mathcal{A}(R, \dot{R})$.

Definition 2 (Maximum Control Amplitude) Given the internally stable standard control loop as in Fig. 1. We call

$$
u_{\max }:=\left(\begin{array}{c}
\sup \left\{\left\|u_{1}\right\|_{\infty} ; \forall r \in \mathcal{A}(R, \dot{R})\right\} \\
\vdots \\
\sup \left\{\left\|u_{n}\right\|_{\infty} ; \forall r \in \mathcal{A}(R, \dot{R})\right\}
\end{array}\right)
$$

the Maximum Control Amplitude.

The definition of admissible reference signals is straightforward from the motivation. The component-wise definition of the maximum control amplitude is motivated by practical reasons, as it enables us to handle hard bounds on each control channel separately. The drawback, however is, that it is unlikely that the maximum output amplitude will be reached in all channels in one "operation mode", i.e. invoked by one reference signal: Consider for instance a system with one reference signal and several control channels, then the maximum amplitude in control channels $i, j$ may be achieved when feeding the system with certain admissible reference signals, say, $r^{(i)}, r^{(j)}$, which are in general different from each other. Thus, when feeding the system with reference signal $r^{(i)}$, control channel $i$ will achieve its maximum amplitude, but $\sup _{t}\left\|u^{j}\right\|=\sup _{t}\left\|h^{j} \star r^{(i)}\right\| \leq$ $\sup _{t}\left\|h^{j} \star r^{(j)}\right\|$ ( " $\star$ " denotes the convolution). This "overestimation" appears because the definition of the maximum control amplitude (1) is not a scalar. Having said this, we restrict ourselves without loss of generality to systems with one control channel only (the generalisation follows by component-wise application of the results obtained in the remainder).

The core of the design procedure to be proposed is that we are able to calculate the maximum control amplitude in a given multivariable control system exactly. For reference purpose, we state the appropriate algorithm:

Algorithm 1 (Calculation of the Maximum Control Amplitude) Given an internally stable LTI control system with $(R, \dot{R})$-admissible reference signal $r=\left[r_{1}, \ldots, r_{k}\right]^{T}$ and scalar control signal $u$. Denote the transfer function from reference to control signal with $H=\left[H_{1}, \ldots, H_{k}\right]$. Let

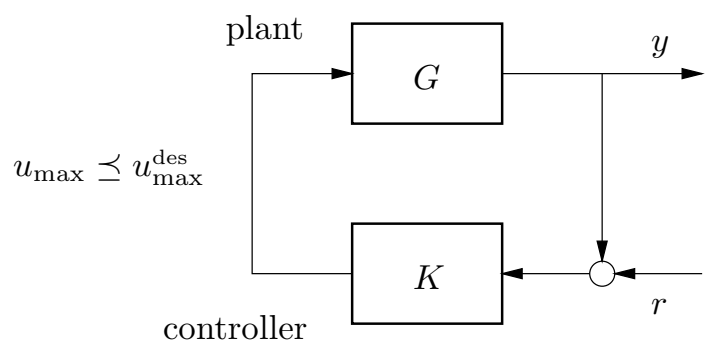

Figure 1: Multivariable control system with constraint control signal $u$, reference signal $r$ and output signal $y$. 
$\left\{h_{i}^{l}\right\}_{l=1}^{\infty}$ the impulse response of $H_{i}$, evaluated on a time grid $\left\{t^{l}\right\}_{l=1}^{\infty}$. Then the maximum control amplitude can be approximated with arbitrary precision by

$$
u_{\max }=\sum_{i=1}^{k} \tilde{u}_{i},
$$

where $\tilde{u}_{i}$ is the solution of the following constrained optimisation problem:

$$
\begin{aligned}
& \tilde{u}_{i}=\max _{r_{i}^{l}}\left|\sum_{l=0}^{\infty} h_{i}^{l} r_{i}^{l}\right| \\
& \text { s.th. } \quad-R_{i} \leq \quad r_{i}^{l} \quad \leq R_{i}, \quad \forall l \geq 0, \\
& -\dot{R}_{i} \leq \quad \frac{r_{i}^{l+1}-r_{i}^{l}}{t^{l+1}-t^{l}} \quad \leq \dot{R}_{i}, \quad \forall l \geq 0 .
\end{aligned}
$$

where $\left\{r_{i}^{l}\right\}_{l=1}^{\infty}$ is the reference signal $r_{i}$, evaluated on the time grid $\left\{t^{l}\right\}_{l=1}^{\infty}$.

Remark 1 Alg. 1 is an infinite optimisation problem with a nonlinear objective (due to the absolute value). It can, however, be reduced to a Linear Program. This is discussed in detail in [18].

In the multivariable case with $n$ control signals, we can apply Alg. 1 for each component separately (cf. the discussion after Def. 2).

\section{Loop Shaping of Systems with Constraints}

Algorithm 1 enables us to calculate the maximum control amplitude of a control system, when the external signal, i.e. the reference signal, is admissible (with given constraints). We will exploit this to extend the $\mathcal{H}_{\infty}$ Loop Shaping Design Procedure, see [19] and Fig. 2, to the case of constraint control signals:

Algorithm 2 (Extended Loop Shaping Design Procedure) Given a (multivariable) plant G, restrictions $R, \dot{R} \succeq 0$ for the reference signal and a desired maximum control amplitude $u_{\max }^{\text {des }} \succ 0$ for all control channels. We will perform $\mathcal{H}_{\infty}$ Loop Shaping in the following way:

1. Choice of design weights $W_{1}, W_{2}$ and performance factor $f \geq 1$.

2. Controller-design for the shaped plant and calculation of the stability margin $\epsilon$.

3. Calculation of the final controller (including the weights).

4. Decide whether the design-objectives are fulfilled or not:

- Is the stability margin $\epsilon$ large enough?

- Are the performance-objectives fulfilled?

- Does $u_{\max } \preceq u_{\max }^{\text {des }}$ hold for all $(R, \dot{R})$-admissible reference signals?

If not, adjust weights $W_{1}, W_{2}$ (and/or performance factor $f$ ) and go to step (1).

The additional check in step 4, if the desired bound on the control signal hold or not, a-posteriori of nature, is the basic difference to classical Loop Shaping. We will discuss the question, how to choose or adjust the design weights in order to accomplish our aim in the next section. 


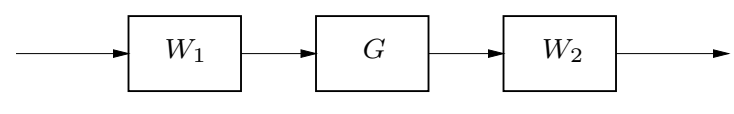

a) plant shaping via $W_{1,2}$

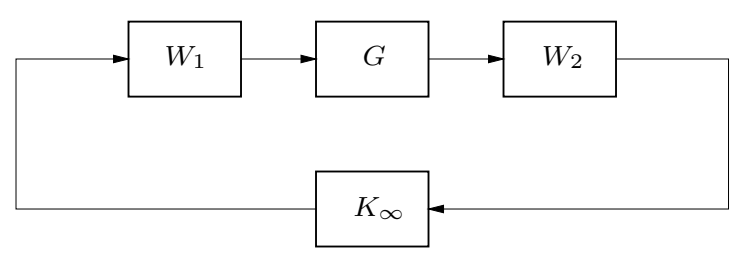

b) $H_{\infty}$ controller design

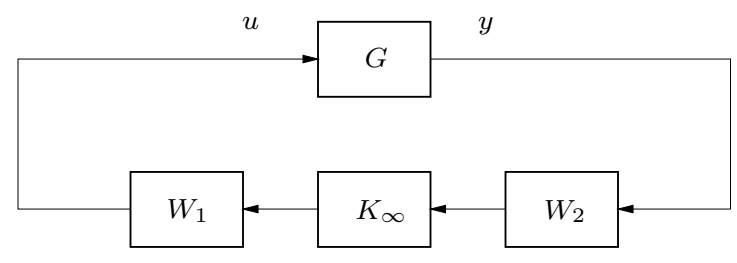

c) final controller

Figure 2: $\mathcal{H}_{\infty}$ Loop Shaping and controller design in three steps.

\section{Adjustment of the Design Weights}

We discuss the question that we left open in the last section: after a first choice of the design weights, our design objectives are usually not fulfilled - the weights have to be adjusted. In the case of a too small stability margin $\epsilon$ the strategy is clear from the classical Loop Shaping: because there is no explicit relation known between achieved stability margin and weights, we have to examine the singular values of the shaped plant and the achieved open loop. In the frequency range with a significant difference, our weights are incompatible with the plant and have to be adjusted. We will not discuss these recommendations further on. Detailed case studies for the classical case are described for example in [19, 20, 21, 22].

In the following we discuss the remaining open question: is there a strategy for correct and systematic adjustment of the weights, when the maximum control signal is still too large after a loop shaping step? The intuitive answer to this question is to pick the next controller (i.e. weight) in such a way that the gain of the transfer function $H$ is lower (this is in fact used in [19] for instance). Note, however, that the norm, induced by the (operator-) $\mathcal{H}_{\infty}$ norm is the 2-norm (in the signal space), in which we are not interested as we have equipped the signal space with the $\infty$-norm instead. However, an explicit relation between $\mathcal{H}_{\infty}$ norm of the transfer function and $\infty$-norm of the signals will be given in (6).

Within Loop Shaping, we work on the singular values of different interesting transfer functions. Thus we are interested in a relation between the singular values of the design weights $W_{1}, W_{2}$ and the maximum control amplitude $u_{\text {max }}$.

In the following, we will need the following result:

Theorem 1 ([23]) Let $H$ be a asymptotically stable and strictly proper transfer function, then

$$
\|H\|_{\infty} \leq\|h\|_{1} \leq 2 \cdot\|H\|_{N}
$$


holds, where $\|\cdot\|_{N}$ denotes the nuclear norm $\|H\|_{N}:=\sum_{j=1}^{n} \sigma_{j}(H)$ and $\sigma_{1} \geq \sigma_{2} \geq \cdots \geq \sigma_{n}>0$ are the Hankel singular values (see [23] or [22, Sec.10.2.2]) in descending order.

Because of the component-wise definition of the maximum control amplitude in Def. 2, we restrict our following examinations to the case of a single control variable, i.e. the transfer function $H$ is MISO. The generalisation follows by component-wise usage. We then have the following:

Corollary 1 (Upper bound for maximum control amplitude (strictly proper case)) Let $H$ be an asymptotically stable and strictly proper transfer function with McMillan degree $n$, input $r$, output $u$ and impulse response $h$. Then the following inequality holds:

$$
\|u\|_{\infty} \leq 2 n \cdot\|H\|_{\infty} \cdot\|r\|_{\infty}
$$

Proof. Let $\|h\|_{1}$ denote the 1-norm of the impulse response. Combining inequality $\|u\|_{\infty} \leq$ $\|h\|_{1}\|r\|_{\infty}$ with (3), we have:

$$
\|u\|_{\infty} \leq\|h\|_{1} \cdot\|r\|_{\infty} \leq 2 \cdot\|H\|_{N} \cdot\|r\|_{\infty}=2 \cdot \sum_{j=1}^{n} \sigma_{j}(H) \cdot\|r\|_{\infty} .
$$

We observe, that $\sigma_{1}(H)=\|H\|_{H}$ (the Hankel norm), for which $\|H\|_{H} \leq\|H\|_{\infty}$ holds [22, Sec.10.2.3] and $\sigma_{1}(H) \geq \sigma_{j}(H)$. Hence, (4) degenerates to

$$
\|u\|_{\infty} \leq 2 n \cdot \sigma_{1}(H) \cdot\|r\|_{\infty} \leq 2 n \cdot\|H\|_{\infty} \cdot\|r\|_{\infty},
$$

as claimed.

We move on to extending this result to the case of proper transfer functions:

Corollary 2 (Upper bound for maximum control amplitude) Let $H$ be an asymptotically stable transfer function with McMillan degree $n$, state space realization $H \stackrel{s}{=}(A, B, C, D)$, input $r$, output $u$ and and impulse response $h$. Denote moreover $D=\left[d_{1}, \ldots, d_{n}\right]^{T}$ and $d:=\max _{i}\left|d_{i}\right|$. Then the following inequality holds:

$$
\|u\|_{\infty} \leq\left(2\|H\|_{\infty}+3 d\right) \cdot n \cdot\|r\|_{\infty} .
$$

Proof. Let $\tilde{H}: \stackrel{s}{=}(A, B, C, 0), \tilde{D}: \stackrel{s}{=}(0,0,0, D)$, then $H=\tilde{H}+\tilde{D}$ and $h(t)=\tilde{h}(t)+D \delta(t)$, with $\delta(t)$ the $\delta$-impulse. Hence:

$$
\begin{aligned}
\|h\|_{1} & =\sum_{i=1}^{n} \int_{0}^{\infty}\left|h_{i}(t)\right| d t=\sum_{i=1}^{n} \int_{0}^{\infty}\left|h_{i}(t)-d_{i} \delta(t)+d_{i} \delta(t)\right| d t \\
& \leq \sum_{i=1}^{n}\left(\int_{0}^{\infty}\left|h_{i}(t)-d_{i} \delta(t)\right| d t+\int_{0}^{\infty}\left|d_{i} \delta(t)\right| d t\right) \\
& \leq \sum_{i=1}^{n} \int_{0}^{\infty}|\tilde{h}(t)| d t+n \max _{i}\left|d_{i}\right|=\|\tilde{h}(t)\|_{1}+n d \\
\text { and }\|u\|_{\infty} & \leq\|h\|_{1}\|r\|_{\infty} \leq\left[\|\tilde{h}\|_{1}+n d\right]\|r\|_{\infty} \leq\left[2 n\|\tilde{H}\|_{\infty}+n d\right]\|r\|_{\infty} \\
& =\left[2 n\|H-\tilde{D}\|_{\infty}+n d\right]\|r\|_{\infty} \leq 2 n\|H\|_{\infty}\|r\|_{\infty}+3 n d\|r\|_{\infty}
\end{aligned}
$$

So far, we derived an explicit relation between the $\mathcal{H}_{\infty}$-norm of the transfer function $H$ (i.e. the maximum over all frequencies of the largest singular value) and the maximum control amplitude. 
The last question to answer is, if there exists a relation between the singular values of the weights and those of the transfer function $H$ ? This discussion is rather standard, cf. for instance [19, Ch.6], we state it for reading convenience:

Using $H=(I-K G)^{-1} K, K=W_{1} K_{\infty} W_{2}$ (cf. Figs. 1 and 2) and denoting $\bar{\sigma}$ and $\underline{\sigma}$ the largest and smallest singular value respectively, we obtain

$$
\begin{aligned}
\bar{\sigma}(H)=\bar{\sigma}\left((I-K G)^{-1} K\right) & \leq 1 / \underline{\sigma}(I-K G) \bar{\sigma}(K) \\
& \approx \bar{\sigma}(K) \quad \text { at freq. with } \bar{\sigma}(K G) \ll 1) \\
& =\bar{\sigma}\left(W_{1} K_{\infty} W_{2}\right) \\
& \leq \bar{\sigma}\left(W_{1}\right) \bar{\sigma}\left(K_{\infty}\right) \bar{\sigma}\left(W_{2}\right) .
\end{aligned}
$$

Obvious from (8), decreasing the weights decreases the transfer function $H$ (in terms of the largest singular value). As the $\mathcal{H}_{\infty}$-norm of $H$ appears at high frequencies (where the open loop gain is low), the restriction on certain frequencies (7) causes no problems. As the control loop is internally stable, the transfer function $H$ is asymptotically stable. Hence, applying (6), we observe that decreasing the $\mathcal{H}_{\infty}$-norm of the weights decreases an upper bound for the maximum control amplitude. Within Loop Shaping, this has the following consequences:

Suppose, the maximum control amplitude is too high after a Loop Shaping step. We then have to decrease the maximum singular value of the design weights in the frequency range where the $\mathcal{H}_{\infty}$-norm of $H$ appears. In the case of a too low maximum control signal, we have to increase the maximum singular value in that frequency range. We point out, that this does only affect an upper bound for the maximum control amplitude. In general, there might be much space between the both sides of (6). We only use it as a guideline for the adjustment of the weights in the "correct direction" and in the correct frequency range. The practical value of this guideline is shown in the example.

Remark 2 So far, we discussed a guideline for weight adjustment for systems with one control channel. In the case of several control channels, the transfer functions from all reference signals to each control channel $u_{i}$ separately has to be examined as discussed above.

Remark 3 Eqn. (6) is still useful for the proper adjustment of design weights, even when we leave the (Loop Shaping-) framework we are using in this work. Describing an upper bound, it is independent of the restrictions on the reference signals' rate or the exact computability of the maximum control amplitude.

\section{Example: Control of the Vertical Dynamics of an Aircraft}

We study a multivariable continuous time plant, an aircraft model, examined in great detail in [19]. The plant has three inputs and outputs, two complex conjugate pole pairs and a pole in the origin. It can be represented by the following state space model:

$$
G(s) \stackrel{s}{=}\left[\begin{array}{ccccc|ccc}
0 & 0 & 1.1320 & 0 & -1 & 0 & 0 & 0 \\
0 & -0.0538 & -0.1712 & 0 & 0.0705 & -0.1200 & 1 & 0 \\
0 & 0 & 0 & 1 & 0 & 0 & 0 & 0 \\
0 & 0.0485 & 0 & -0.8556 & -1.0130 & 4.4190 & 0 & -1.6650 \\
0 & -0.2909 & 0 & 1.0532 & -0.6859 & 1.5750 & 0 & -0.0732 \\
\hline 1 & 0 & 0 & 0 & 0 & 0 & 0 & 0 \\
0 & 1 & 0 & 0 & 0 & 0 & 0 & 0 \\
0 & 0 & 1 & 0 & 0 & 0 & 0 & 0
\end{array}\right] .
$$

Throughout the example, we determine suboptimal controllers $(f=1.1)$. McFarlane \& Glover demand, additionally to performance and stability objectives, the following component-wise bounds 
on the control signal $u$ :

$$
\left|u_{1}(t)\right|<40, \quad\left|u_{2}(t)\right|<10, \quad\left|u_{3}(t)\right|<40, \quad \forall t \geq 0 .
$$

To solve this problem with the proposed extension of the Loop Shaping procedure, we restrict the reference signal by the following values:

$$
R=[1,1,1]^{T}, \quad \dot{R}=[5,11,3]^{T} .
$$

\subsection{Analysis of the McFarlane \& Glover Design}

We start up with the design [19, Sec. 7.4.3, design 2] based on the following diagonal weight $W(s)=\operatorname{diag}\left\{w_{1}(s), w_{2}(s), w_{3}(s)\right\}$ with:

$$
w_{1}(s)=w_{3}(s)=24 \cdot w_{c}, \quad w_{2}(s)=12 \cdot w_{c}, \quad w_{c}(s)=\frac{s+0.4}{s}
$$

at the plant output. The usage of one diagonal weight ensures a better oversight during Loop Shaping. Therefore, we restrict ourselves to this class of weights. The gain increases the open loop and thus increases the $0 d B$ crossover frequency. The integral action will improve the low frequency performance. Looking at the singular values of the unshaped plant (see Fig. 3, upper plot), the zeros at -0.4 limit the integrator to the low frequency range, so that a too high roll-off rate near the crossover frequency is prevented. This would cause poor robustness properties (i.e. a small stability margin) or even instability (known from Bode's Gain-Phase relations). The weight $W$ leads to a eleventh order controller $K$ and the resulting system has the following maximum control amplitude:

$$
u_{\max }=[26.53,10.49,61.42]^{T}
$$

for all admissible reference signals obeying (11). The stability margin is $\epsilon=0.38$ and Fig. 3 shows singular values ${ }^{2}$ of some closed loop functions. We observe from the above computed value $u_{\max }$, compared to (10), that we can "effort" much more in the first control channel, while the amplitude in the second one is slightly, and in the third one is quite too large. Aim of a proper weight adjustment will be, according to the recommendations in Sec. 4, to increase the gain of the transfer function from (all) reference signals to the first control channel, and to decrease gain of the transfer functions from (all) reference signals to the second and third control channel respectively. These gains are reported in Fig. 4.

\subsection{Adjustment of the Weights}

Now, we adjust the weight $W$ in order to achieve the meet maximum control amplitude. According to the results derived in Sec. 4, we are looking at the singular values in Fig. 4. We see, that they achieve their maximum at frequencies around $10 \mathrm{rad} / \mathrm{s}$. As motivated above, we have to increase the first and to decrease the other ones by proper adjustment of the weight $W$. Hence, we increase the first diagonal entry $w_{1}$ and decrease the other two entries of the weight $W$ in this frequency

\footnotetext{
${ }^{2}$ Throughout this example, we only show largest and smallest singular values.
} 

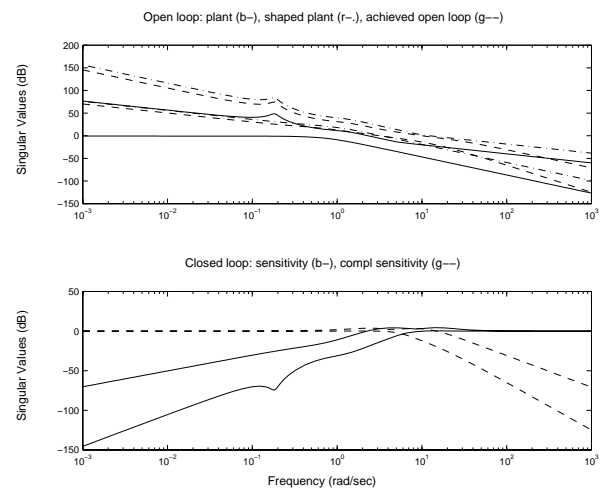

Figure 3: McFarlane \& Glover design: Upper plot: singular values of plant (solid), shaped plant (dash dotted) and achieved open loop (dashed). Lower plot: singular values of sensitivity (solid) and complementary sensitivity (dashed).
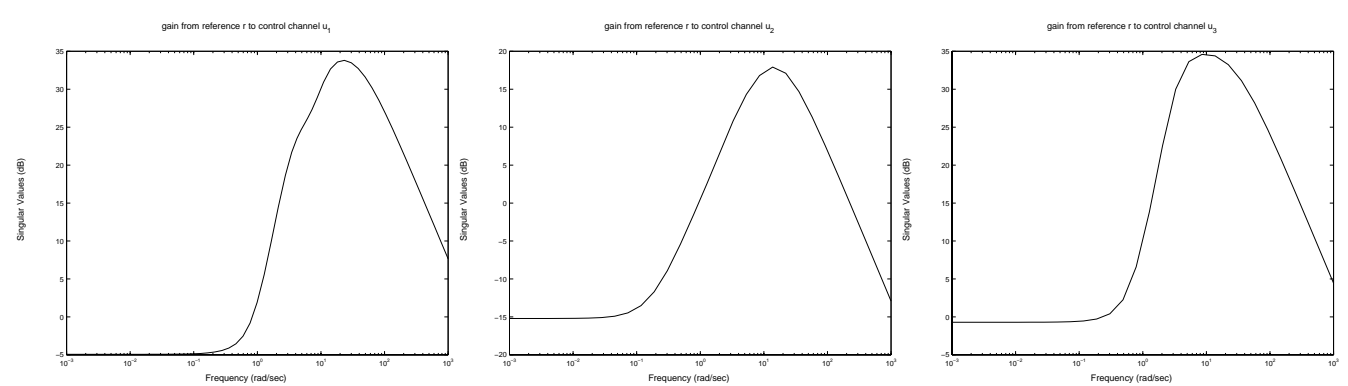

Figure 4: McFarlane \& Glover design: gains of the transfer functions from reference signal (vector) to control channels 1-3 separately (left to right). 

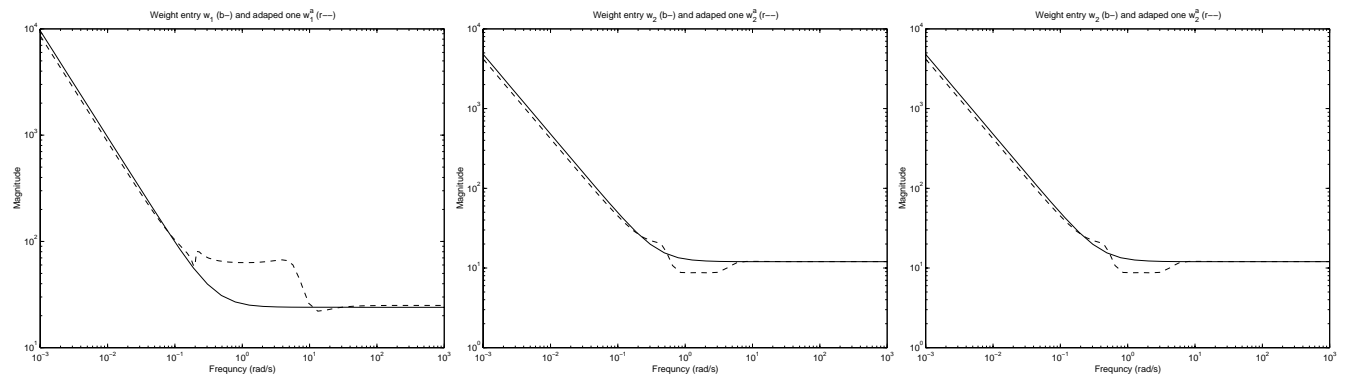

Figure 5: Three diagonal entries (left to right) of the McFarlane and Glover weight $W$ (solid) and the adjusted weight $W^{a}$ (dashed): The first entry was increased while the other ones have been decreased in the frequency range of interest.

range respectively. The adjusted weight is $W^{a}=\operatorname{diag}\left\{w_{1}^{a}, w_{2}^{a}, w_{3}^{a}\right\}$, see also Fig. 5, with:

$$
\begin{aligned}
& w_{1}^{a}=\frac{25 s^{5}+216.9 s^{4}+2425 s^{3}+401.1 s^{2}+102.7 s+13.76}{s^{5}+5.781 s^{4}+38.81 s^{3}+1.135 s^{2}+1.597 s}, \\
& w_{2}^{a}=\frac{12.02 s^{5}+52.87 s^{4}+219.5 s^{3}+157.8 s^{2}+101.3 s+21.4}{s^{5}+4.754 s^{4}+21.4 s^{3}+7.626 s^{2}+5.104 s}, \\
& w_{3}^{a}=\frac{24.2 s^{5}+184.9 s^{4}+1761 s^{3}+633.6 s^{2}+298.4 s+0.3928}{s^{5}+19.2 s^{4}+382 s^{3}+77.01 s^{2}+0.1529 s} .
\end{aligned}
$$

Using the adjusted weight $W^{a}$, we obtain a maximum control amplitude of:

$$
u_{\max }=[39.06,10.00,39.62]^{T}
$$

and achieve a stability margin of $\epsilon^{a}=0.30$. The resulting controller $K^{a}$ is of order 35 and can easily obtained using Matlab's $\mu$ Toolbox command ncfsyn.

Our design objectives regarding the constraint control variable are fulfilled! Fig. 6 shows the singular values of the control system made up with controller $K^{a}$. The singular values of the transfer functions to the single components of the control signal, have been adjusted correctly in the frequency range in question, as reported in Fig. 7: increased from $34 d B$ to $37 d B$ for the first control channel and decreased from $17.5 d B$ to $17 d B$ (35dB to $27 d B$ ) in the second (third) control channel.

\subsection{Simulation Studies}

We simulate both closed loop systems (employing controllers $K$ and $K^{a}$ respectively), including a saturation nonlinearity, and assuming the presence of a white band limited noise with power $10^{-6}$ at the plant output (cf. Fig. 1). We stimulate both systems with an $\left([1,1,1]^{T},[5,11,3]^{T}\right)$-admissible reference signal. The reference/output signals and control signals are reported in Figs. 8 and 9 respectively. We observe, that the control system including controller $K$ runs into saturation two times (in the third control channel), while the control system made up with controller $K^{a}$ does not. This fulfills our expectation, as we reported a much too large third control amplitude in the earlier analysis.

We observe no significant difference when tracking output channels 2 and 3 . The tracking of channel 1, however, has improved when applying the adjusted controller (or, in turn this means that running into saturation degrades the performance of the system with the original controller being involved). Similar behaviour can be observed in other simulation studies. 

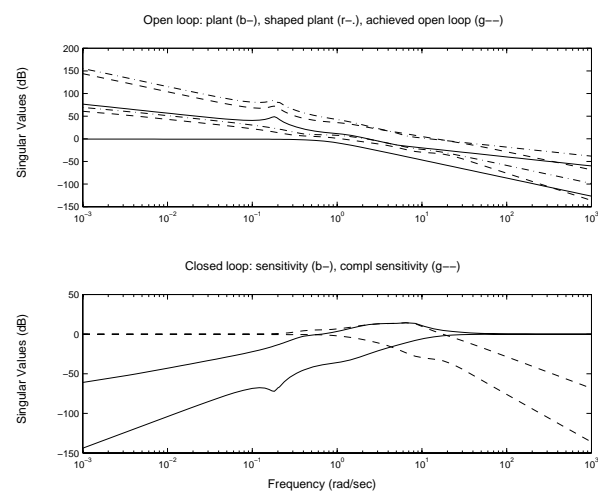

Figure 6: Design with adjusted weights: Upper plot: singular values of plant (solid), shaped plant (dash dotted) and achieved open loop (dashed). Lower plot: singular values of sensitivity (solid) and complementary sensitivity (dashed).
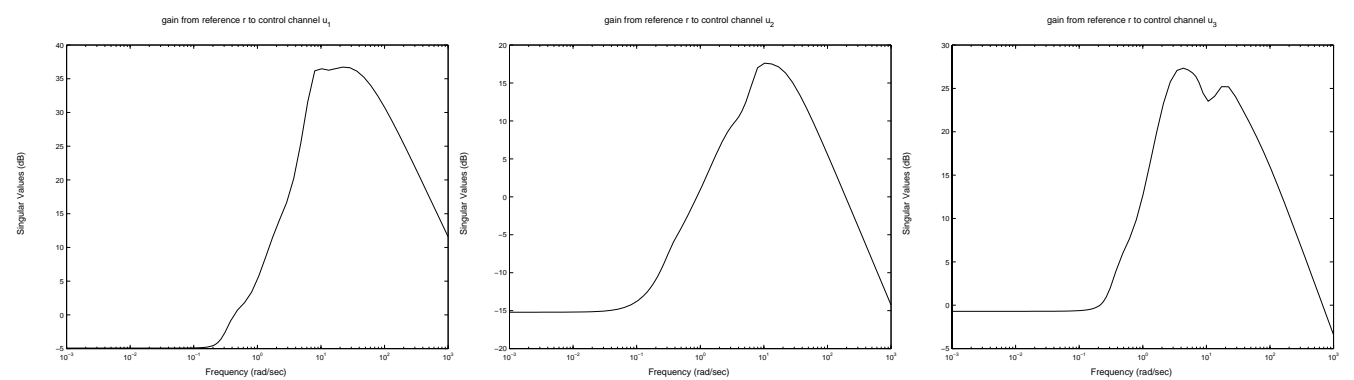

Figure 7: Adjusted design: gains of the transfer functions from reference signal (vector) to control channels 1-3 separately (left to right).

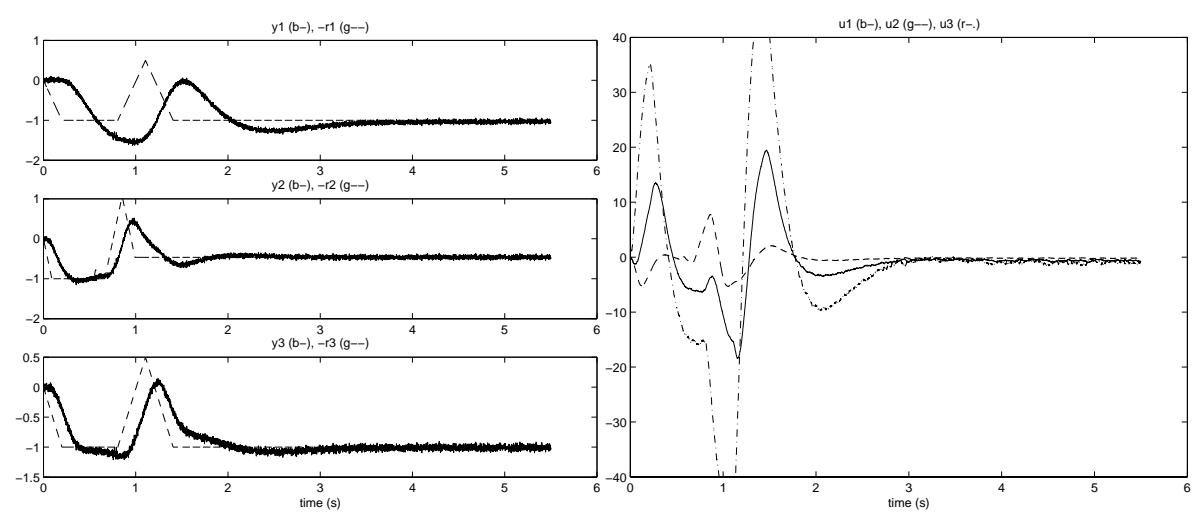

Figure 8: Simulation of control system with controller $K$. Left: reference (dashed) and output signals (solid). Right: control signals 1 (solid), 2 (dashed) and 3 (dash-dotted). 


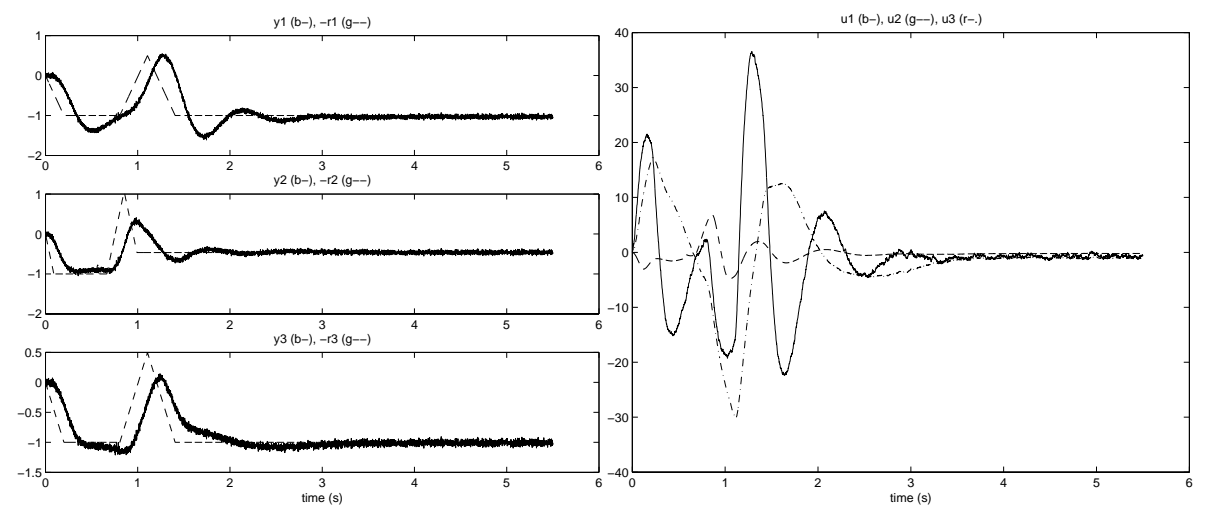

Figure 9: Simulation of control system with adjusted controller $K^{a}$. Left: reference (dashed) and output signals (solid). Right: control signals 1 (solid), 2 (dashed) and 3 (dash-dotted).

\section{Conclusions and Future Works}

We studied the control of multivariable control systems with hard bounded control signals. One main point within the extended $\mathcal{H}_{\infty}$ Loop Shaping procedure was the calculation of the maximum control amplitude for a set of admissible reference signals. The other main point was the systematic adjustment on the weights with respect to the control signals bound by deriving an explicit relation between design weight and maximum control amplitude. The proposed method and guideline have been illustrated with an example.

An open question is how to guarantee the control signal within the suggested bounds for an uncertain plant - until now, this is only guaranteed for the nominal plant without any noise. However, the simulation studies in this work showed sufficient behaviour of the control signal when facing output error noise; other simulation studies (see [24]) showed a satisfactory behaviour when having uncertain (real) parameters. Ongoing work, for instance in [17] investigates this question for single-input single output uncertain plants.

\section{Acknowledgement}

This work was partly supported by the German research council (DFG) while the author was with Paderborn University, Paderborn, Germany. Valuable discussions with F. Gausch and A. Hofer are gratefully acknowledged .

\section{References}

[1] A. Saberi, A. A. Stoorvogel, and P. Sannuti. Control of Linear Systems with Regulation and Input Constraints. Communications and Control Engineering. Springer Verlag, London, UK, 2000 .

[2] S. Tabouriech and G. Garcia, editors. Control of Uncertain Systems with Bounded Inputs, volume 227 of Lecture Notes in Control and Information Science. Springer Verlag, London, UK, 1997.

[3] D. S. Bernstein and A. N. Michel. A chronological bibliography on saturating actuators. Int. J. of Robust and Nonlinear Control, 5:375-380, 1995. Special Issue Saturating Actuators. 
[4] A. A. Stoorvogel and A. Saberi. Special issue on control problems with constraints. Int. J. of Robust and Nonlinear Control, 9(10), 1999.

[5] M. V. Kothare, V. Balakrishnan, and M. Morari. Robust Constrained Model Predictive Control using Linear Matrix Inequalities. Automatica, 32(10):1361-1379, October 1996.

[6] C. Barbu, R. Reginatto, A. R. Teel, and L. Zaccarian. Anti-windup for exponentially unstable linear systems with inputs limited in magnitude and rate. In Proc. of the American Control Conference, pages 1230-1234, Chicago, IL, USA, 2000.

[7] M. V. Kothare, P. J. Campo, M. Morari, and C. N. Nett. A unified framework for the study of Antiwindup designs. Automatica, 30(12):1869-1883, December 1994.

[8] E. G. Gilbert and K. T. Tan. Linear systems with state and control constraints: The theory and applications of maximal output admissible sets. IEEE Trans. on Automatic Control, 36(9):1008-1020, September 1991.

[9] E. D. Sontag. An algebraic approach to bounded controllability of linear systems. Int. J. of Control, 39(1):181-188, January 1984.

[10] H. J. Sussmann, E. D. Sontag, and Y. Yang. A general result on the stabilization of linear systems using bounded controls. IEEE Trans. on Automatic Control, 39(12):2411-2425, December 1994.

[11] A. Saberi, J. Han, and A. A. Stoorvogel. Constrained stabilization problems for linear plants. In Proc. of the American Control Conference, pages 4393-4397, Chicago, IL, USA, 2000.

[12] J. A. De Dona, R. Moheimani, and G. C. Goodwin. Robust combined PLC/LHG controller with allowed over-saturation of the input signal. In Proc. of the American Control Conference, pages 750-754, Chicago, IL, USA, 2000.

[13] N. Dourdoumas. Prinzipien zum Entwurf linearer Regelkreise mit Beschränkungen - eine Einführung. Automatisierungstechnik, 35(8):301-309, August 1987.

[14] R. W. Reichel. Entwurf von Regelsystemen mit Begrenzungen. Automatisierungstechnik, 34(11):446-453, November 1986.

[15] D. Holtgrewe. Entwurf von Mehrfachsystemen mit beschränkten Systemgrössen. PhD thesis, Dept of EE, University of Paderborn, 33095 Paderborn, Germany, 1992.

[16] W. Reinelt. Design of optimal control systems with bounded control signals. In Proc. of the European Control Conference, Porto, Portugal, September 2001.

[17] W. Reinelt and M. Canale. Robust control of SISO systems subject to hard input constraints. In Proc. of the European Control Conference, Porto, Portugal, September 2001.

[18] W. Reinelt. Maximum Output Amplitude of Linear Systems for certain Input Constraints. In Proc. of the IEEE Conference on Decision and Control, pages 1075-1080, Sydney, Australia, December 2000.

[19] D. C. McFarlane and K. Glover. Robust Controller Design Using Normalized Coprime Factor Plant Description. Number 138 in Lecture Notes in Control and Information Science. Springer Verlag, Berlin, Germany, 1989. 
[20] J. Feng and M. C. Smith. When is a controller optimal in the sense of $H_{\infty}$ Loop-Shaping? IEEE Trans. on Automatic Control, 40(2):2026-2039, December 1995.

[21] R. A. Hyde. The Application of Robust Control to VSTOL Aircraft. PhD thesis, Dept of Engineering, University of Cambridge, Cambridge CB2 1PZ, UK, 1991.

[22] M. Green and D. J. N. Limebeer. Linear Robust Control. Prentice Hall, Englewood Cliffs, NJ, USA, 1995.

[23] K. Glover. Model reduction: A tutorial on Hankel-norm methods and lower bounds on $L^{2}$ errors. In Proc. of the 10th IFAC World Congress, München, Germany, 1987.

[24] W. Reinelt. $\mathcal{H}_{\infty}$ Loop Shaping for Systems with Hard Bounds. In Proc. of the Int Symp on Quantitative Feedback Theory and Robust Frequency Domain Methods, pages 89-103, Durban, South Africa, August 1999. 